\title{
Clinical Reasoning: Progressive cognitive decline, cerebellar ataxia, recurrent myoclonus, and epilepsy
}

Fei Xiao, MD, Jingchuan Fan, BS, Jiaze Tan, BS, and Xue-feng Wang, MD

Neurology ${ }^{\circledR}$ 2018;90:e1827-e1831. doi:10.1212/WNL.0000000000005528
Correspondence

Dr. Wang

Isxiaof@sina.com

\section{Section 1}

A 23-year-old woman was admitted to our department due to recurrent attacks of myoclonus and generalized tonic-clonic seizures, progressive intellectual disability, and gait instability. Her family history was unremarkable. The patient's growth and developmental milestones in childhood were normal. At the age of 12 years, she initially complained of lower limb fatigue and slow running. One year later, she began to exhibit a progressive decline in memory and understanding. She subsequently became introverted. At 15 years of age, her cognitive decline aggravated and bradykinesia and upper limb motor tremor appeared and she had slurred speech. At the age of 18, she had her first generalized tonic-clonic seizure (GTCS) during sleep. Shortly thereafter, myoclonus jerks appeared. Since then, she has experienced recurrent GTCS attacks and myoclonic jerking. Levetiracetam and clonazepam were given, but the seizures and myoclonic jerking remained poorly controlled. At the age of 22 years, she was no longer able to control body balance and often fell while walking and could not perform fine movements such as writing. Her vision and hearing were not impaired.

Physical examination revealed dysarthria and intellectual deterioration. Cognitive testing yielded a Mini-Mental State Examination score of 16/30 and a Montreal Cognitive Assessment score of 12/30. Areas of cognitive dysfunction included orientation, attention and concentration, language, memory, and visuospatial function. Bradykinesia and postural and intention tremor were apparent. Mild weakness (classified as grade 4 according to the Medical Research Council criteria) was detected in all 4 limbs. Muscle tone was decreased, but deep reflexes were exaggerated and a positive bilateral Babinski sign was noticed. The patient exhibited dysmetria on the finger-to-nose test, moderate limb ataxia, and a prominent instability of gait. The gait was wide-based, hypokinetic, and ataxic, with difficulty turning, and the arm swing was significant reduced. Myoclonic jerking in the trunk and limbs could occasionally be noted. Visual acuity and hearing test results were normal. Corneal Kaiser-Fleischer ring was not detected by slitlamp examination. Cherry-red spot and macular degeneration were not found on fundus examination.

\section{Questions for consideration:}

1. In which areas could you localize possible lesions?

2. What is your initial approach to investigation?

\section{GO TO SECTION 2}

\footnotetext{
From the Department of Neurology (F.X., J.T., X.-f.W.), The First Affiliated Hospital of Chongqing Medical University, Chongqing Key Laboratory of Neurology; and Department of Medical Laboratory Technology (.F.), Institute of Life Sciences of Chongqing Medical University, Chongqing, China.

Go to Neurology.org/N for full disclosures. Funding information and disclosures deemed relevant by the authors, if any, are provided at the end of the article.
} 


\section{Section 2}

Cognitive decline was suggestive of cerebral cortex or hemisphere involvement. Myoclonus and GTCS indicated cerebral cortex involvement. Bilateral pyramidal tracts were also affected given the hyperreflexia and bilateral positive Babinski sign. The patient revealed prominent cerebellar involvement showing dysmetria on the finger-to-nose test, limb ataxia, intentional tremor, and decreased muscle tone. In addition, the presence of postural tremor suggested involvement of the extrapyramidal system. Thus, multiple structures of the CNS seem to be affected.

Ancillary investigations performed in the patient are summarized in the table.

\section{Question for consideration:}

1. What would you consider in the differential diagnosis?

Table Ancillary investigations performed

\begin{tabular}{ll}
\hline Investigations & Results \\
\hline $\begin{array}{l}\text { Laboratory examinations } \\
\begin{array}{l}\text { Baseline chemistry } \\
\text { (electrolytes, lactate, renal, liver, creatine kinase, thyroid function) }\end{array}\end{array}$ & Normal \\
\hline Serum ceruloplasmin & Normal \\
\hline Antinuclear antibody profile & Normal \\
\hline $\begin{array}{l}\text { Serum and urine metabolic parameters } \\
\text { (vitamin } \mathbf{B}_{\mathbf{1 2}} \text { folate, organic acids, amino acids, fatty acids) }\end{array}$ & Normal \\
\hline Leukocyte lysosomal enzymes activities & Normal \\
\hline Cerebral MRI & Moderate to severe generalized brain atrophy (figure, A-C) \\
\hline Electroretinogram & Normal \\
\hline $\begin{array}{l}\text { Visual evoked potentials } \\
\text { EEG }\end{array}$ & Normal \\
\hline EMG & $\begin{array}{l}\text { Spikes and slow waves in temporal, occipital, } \\
\text { and parietal lobes }\end{array}$ \\
\hline Nerve conduction & Normal \\
\hline
\end{tabular}




\section{Section 3}

Neurodevelopmental regression was a characteristic feature of the patient. The onset in this young patient was insidious, and symptoms aggravated progressively, indicating the possibility of a genetic or metabolic neurodegenerative disorder. The combination of features with which the patient presented, including a myoclonus/epilepsy that did not respond well to antiepileptic drugs, cerebellar ataxia, and progressive cognitive decline, points toward progressive myoclonus epilepsy (PME). However, various other conditions could also be considered. Treatable systemic conditions, including vitamin $\mathrm{B}_{12}$ deficiency, hypothyroidism, and Wilson disease, were excluded based on the normal laboratory results. Lysosomal disorders usually lead to cognitive regression in juvenile patients and should thus be considered. ${ }^{1}$ However, the results of metabolic screening and lysosomal enzyme analysis were normal. A decline in intelligence can also occur in several subtypes of spinocerebellar ataxia (SCA), including SCA 2, 3, and 6. However, myoclonus and epilepsy are not prominent features of SCA, so SCA was less likely.

PMEs are a group of rare and heterogeneous neurodegenerative disorders defined by the combination of action myoclonus, epileptic seizures, and progressive neurologic decline; they may include ataxia, progressive cognitive decline, neuropathy, and myopathy. PMEs primarily include several specific disorders: Unverricht-Lundborg disease (progressive myoclonus epilepsy type 1 [EPM1]), Lafora disease (EPM2), neuronal ceroid lipofuscinoses (NCLs), myoclonus epilepsy with ragged-red fibers (MERRF), type 3 neuronopathic Gaucher disease, dentatorubral-pallidoluysian atrophy (DRPLA), action myoclonus-renal failure syndrome (EPM4), PME-ataxia syndrome (EPM5), and North Sea PME (EPM6). Due to the clinical similarities, to distinguish different types of PMEs is challenging. ${ }^{2}$ Nevertheless, it is still possible to make a preliminary differential diagnosis based on the subtle differences in the clinical features and routine auxiliary examinations. Visual loss can appear in patients with sialidosis, Lafora disease, or NCLs (except Kufs disease). Sialidosis, Lafora disease, and NCLs show prominent cognitive impairment, whereas patients with EPM1, EPM4, EPM5, and EPM6 exhibit mild or absent cognitive decline. Ataxia is a common symptom of all types of PMEs, but early onset (average less than 5 years of age) indicates EPM5 or EPM6. Elevated creatine kinase indicates EPM6. Increased serum or CSF lactate level suggests MERRF. The presence of proteinuria indicates EPM4. Macular cherry-red spot by funduscopy raise the consideration of sialidosis. In addition, patients with NCLs (except Kufs disease), Lafora disease, or Gaucher disease usually die within a decade of disease onset; lifespan is relatively longer for MERRF, EPM1, and Kufs disease.

As for our patient, visual loss was not presented, and fundus examination was normal, thus sialidosis, Lafora disease, and NCLs (except Kufs disease) were excluded; cognitive decline was marked, therefore EPM1, EPM4, EPM5, and EPM6 were less likely, combined normal levels of urine protein and creatine kinase, EPM4 and EPM6 were excluded. There was no sign of chorea in our patient; although it cannot be excluded, the possibility of DRPLA was relatively lower. Leukocyte lysosomal enzyme (including $\beta$-glucocerebrosidase) activities were normal and the disease course exceeded 10 years, which did not support the diagnosis of neuronopathic Gaucher disease. Although lactate level was normal, MERRF could not be excluded.

Taken together, we speculated that the patient may have one of the following diseases: Kufs disease, EPM1, or MERRF.

\section{Questions for consideration:}

1. What further tests are indicated to narrow the differential diagnosis?

2. What is the final diagnosis?

GO TO SECTION 4 


\section{Section 4}

Muscle and skin biopsies are effective methods for the differential diagnosis of PMEs. Further genetic testing is helpful to confirm the diagnosis. Therefore, we carried out muscle and skin biopsies. We also collected the patient's blood sample. We did not find ragged-red fibers in the muscle. Lafora bodies were not found in the muscle fibers or skin sweat gland cells. Interestingly, in a small amount of muscle fibers, some red granules were revealed by acid phosphatase staining, indicating increased acid phosphatase activity (figure, D). Electron microscopy of muscle and skin samples yielded normal findings. Wright staining of the peripheral blood smear revealed small cytoplasmic vacuoles in a minority of peripheral blood leukocytes (figure, E). Granular osmiophilic deposits (GRODs) were detected in sporadic blood lymphocytes using electron microscopy (figure, F). The histopathologic features did not provide evidence of MERRF or Lafora disease. Increased acid phosphatase activity in skeletal muscle fibers suggested lysosomal dysfunction. The presence of vacuolated lymphocytes and GRODs in the lymphocytes indicated intracellular deposits of abnormal lipopigments. Although similar structures were not found in muscle and skin tissue, NCLs should be considered. Therefore, the diagnosis of Kufs disease should be considered in combination with the clinical manifestation. Further genetic testing may be helpful to confirm the diagnosis.

Although we obtained the histopathologic evidence, and considered the diagnosis of Kufs disease with CLN6 mutation, in order to exclude other rare causes, we performed whole-exome sequencing. Whole-exome sequencing revealed a compound heterozygous mutation in the CLN6 gene: a point mutation c.749G $>\mathrm{C}$ in one allele and a deletion mutation c.794 (exon7) _c.796 (exon7)delCCT in the other allele. The patient's healthy parents were each a carrier of a mutation, but they were not affected. Mutations of CLN6 were identified in variant of lateinfantile NCL (vLNCL) and recessive Kufs disease type $\mathrm{A}^{3,4}$ Patients with vLNCL experience symptom onset at an age between 18 months and 5 years, with loss of vision. Since our patient did not show visual impairment as a teenager, we excluded vLNCL.

\section{Discussion}

Accurate diagnosis of PMEs usually requires tissue biopsy and genetic tests. Advances in genetic testing have made diagnoses easier and more accurate. Microarray and next-generation sequencing panels designed for epilepsy could find already known gene mutations accounting for PMEs syndromes. In
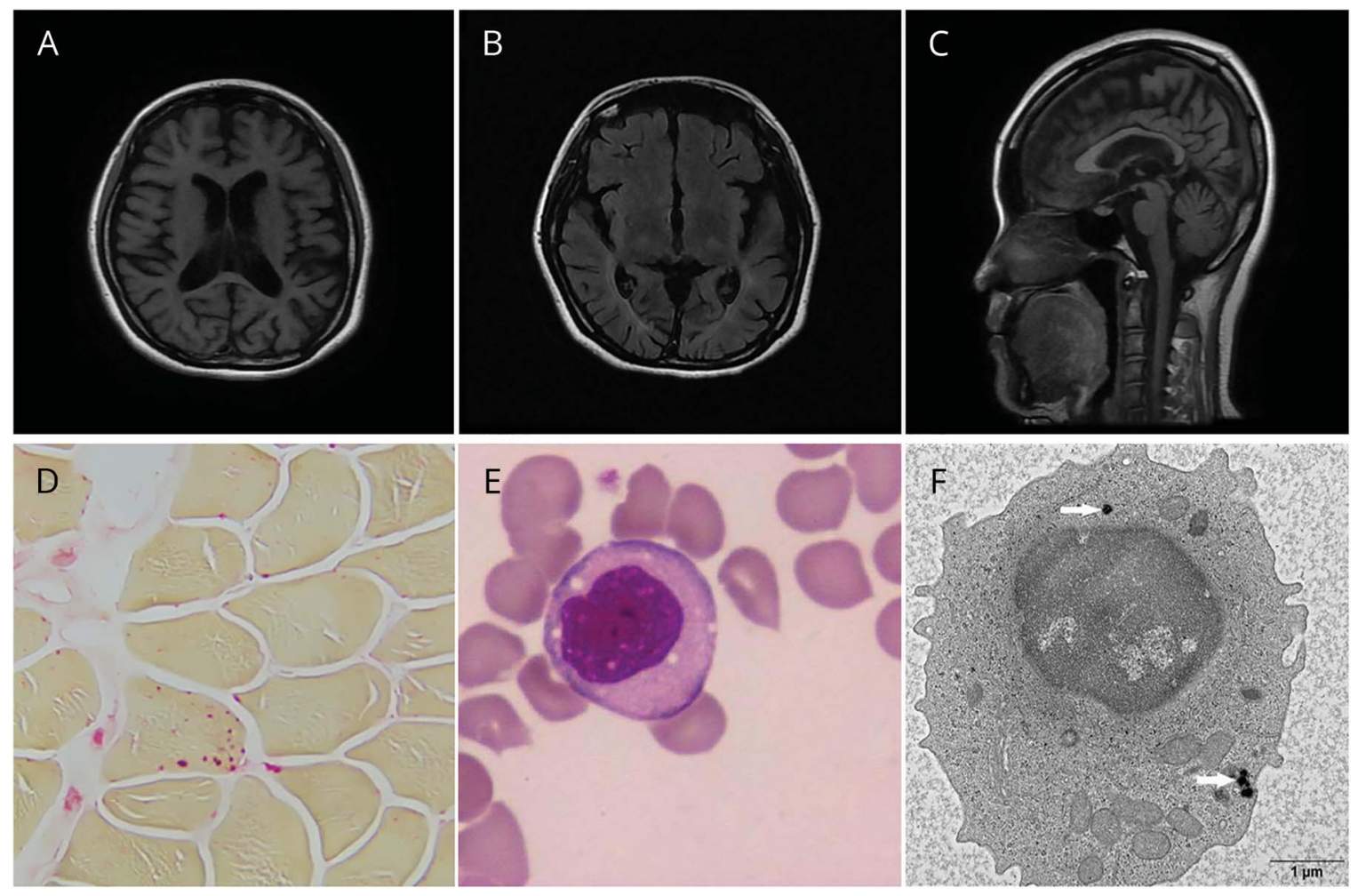

(A) Axial T1-weighted MRI demonstrates dilation of the lateral ventricle and diffuse moderate cerebral atrophy. (B) Axial fluid-attenuated inversion recovery image reveals moderate widespread cerebral atrophy, dilation of the third ventricle, and lateral fissures. (C) Midline sagittal T1-weighted image reveals moderate atrophy of the cerebellar hemisphere. (D) A frozen section of a muscle sample shows several small red granules (acid phosphatase staining positive) in a muscle fiber (acid phosphatase staining, original magnification $\times 400)$. (E) Blood smear reveals several discrete small cytoplasmic vacuoles in a leukocyte (wright staining, original magnification $\times 1,000)$. (F) Lymphocyte ultrastructure detected by electron microscope. The white arrows indicate the granular osmiophilic deposits. Scale bar, $1 \mu \mathrm{m}$. 
addition to detecting known gene mutations, whole-genome sequencing could also display not yet reported and new causative genes and mutations. Kufs disease is the adult-onset form of NCL, which lacks the typical visual impairment seen in other subtypes. ${ }^{4}$ Two different and often overlapping forms have been described: type A, which is characterized by myoclonic epilepsy, ataxia, and dysarthria; and type B, which is characterized by severe cognitive impairment. ${ }^{5}$ Disease onset is usually in the third decade of life but onset in teenagers has also been reported. ${ }^{3,4}$ Two gene mutations have been reported to account for the majority of patients: DNAJC5 mutations lead to the autosomal dominant inherited type Kufs disease and CLN6 mutations cause the autosomal recessive inherited type Kufs disease. ${ }^{6}$ Cerebral MRI usually shows brain atrophy.

NCLs are a group of inherited neurodegenerative disorders characterized by lysosomal lipopigment storage mainly in neurons, and the retina is usually involved (except in the adult form of NCL, Kufs disease $)^{3}$; however, lysosomal lipopigment has also been reported among extracerebral tissues, including lymphocytes, skin, rectum, and skeletal muscle. Cytoplasmic vacuoles of lymphocytes may also be revealed in a blood smear examination using light microscopy. ${ }^{7}$ Increased acid phosphatase reaction in muscle fibers has been found in infantileonset NCL. ${ }^{8}$ The neuropathologic diagnosis of NCLs requires the presence of deposits that accumulate in fingerprint, curvilinear, or granular osmiophilic patterns, as shown by ultrastructural examination. Extraneural tissue biopsies from muscles, skin, and rectum and leukocyte examination can also be used for the diagnosis of NCLs. ${ }^{9}$ The morphologic evaluation of skin biopsies in Kufs disease has been reported to show normal findings, ${ }^{4,9}$ as was the case in our patient. GRODs have been detected in the blood lymphocytes of patients with Kufs disease. ${ }^{7}$ Genetic testing may be helpful to confirm the diagnosis. There is no treatment available to prevent disease progression in Kufs disease, and the treatment is currently symptomatic and palliative. The prognosis is poor because the neurologic function will inevitably progressively deteriorate.

The treatment of PMEs includes the management of seizures and myoclonus and palliative, supportive, and rehabilitative measures. Traditional antiepileptic drugs used for the treatment of PMEs are valproate, clonazepam, and phenobarbital; however, both myoclonus and seizures are usually poorly controlled and tend to be refractory. When treating PMEs, particular care should be paid to avoid drugs known to aggravate myoclonus, including vigabatrin, carbamazepine, phenytoin, gabapentin, and lamotrigine. ${ }^{10}$ Valproate is prohibited in MERRF.

\section{Author contributions}

Fei Xiao: acquisition of data, study concept and design, writing of the manuscript. Jingchuan Fan: electron microscopy analysis of the specimens. Jiaze Tan: processing muscle and skin samples and staining. Xue-feng Wang: study concept and design, critical revision of manuscript for intellectual content.

\section{Study funding}

Study funded by grant 2012-649 from the National Key Clinical Specialist Construction Programmes of China and grant 81301110 from the National Natural Science Foundation of China.

\section{Disclosure}

The authors report no disclosures relevant to the manuscript. Go to Neurology.org/N for full disclosures.

\section{References}

1. Allen NM, Shahwan A, Madigan C, et al. Clinical Reasoning: Juvenile neurocognitive decline: a "snaky" diagnosis. Neurology 2015;85:e170-e174.

2. Kälviäinen R. Progressive myoclonus epilepsies. Semin Neurol 2015;35:293-299.

3. Arsov T, Smith KR, Damiano J, et al. Kufs disease, the major adult form of neuronal ceroid lipofuscinosis, caused by mutations in CLN6. Am J Hum Genet 2011;88: 566-573.

4. Canafoglia L, Gilioli I, Invernizzi F, et al. Electroclinical spectrum of the neuronal ceroid lipofuscinoses associated with CLN6 mutations. Neurology 2015;85: 316-324.

5. Berkovic SF, Carpenter S, Andermann F, Andermann E, Wolfe LS. Kufs' disease: a critical reappraisal. Brain 1988;111:27-62.

6. Cotman SL, Karaa A, Staropoli JF, Sims KB. Neuronal ceroid lipofuscinosis: impact of recent genetic advances and expansion of the clinicopathologic spectrum. Curr Neurol Neurosci Rep 2013;13:366.

7. Moro F, Gismondi F, Pezzini F, Santorelli FM, Simonati A. Clinical, ultrastructural, and molecular studies in a patient with Kufs disease. Neurol Sci 2014;35:605-607.

8. Carpenter S, Karpati G. Lysosomal storage in human skeletal muscle. Hum Pathol 1986;17:683-703.

9. Gelot A, Maurage CA, Rodriguez D, et al. In vivo diagnosis of Kufs' disease by extracerebral biopsies. Acta Neuropathol 1998;96:102-108.

10. Michelucci R, Pasini E, Riguzzi P, Andermann E, Kälviäinen R, Genton P. Myoclonus and seizures in progressive myoclonus epilepsies: pharmacology and therapeutic trials. Epileptic Disord 2016;18:145-153. 


\title{
Neurology
}

\author{
Clinical Reasoning: Progressive cognitive decline, cerebellar ataxia, recurrent \\ myoclonus, and epilepsy \\ Fei Xiao, Jingchuan Fan, Jiaze Tan, et al. \\ Neurology 2018;90;e1827-e1831 \\ DOI 10.1212/WNL.0000000000005528
}

\section{This information is current as of May 14, 2018}

\section{Updated Information \& Services}

References

Subspecialty Collections

Permissions \& Licensing

Reprints including high resolution figures, can be found at: http://n.neurology.org/content/90/20/e1827.full

This article cites 10 articles, 2 of which you can access for free at: http://n.neurology.org/content/90/20/e1827.full\#ref-list-1

This article, along with others on similar topics, appears in the following collection(s):

Gait disorders/ataxia

http://n.neurology.org/cgi/collection/gait_disorders_ataxia Metabolic disease (inherited)

http://n.neurology.org/cgi/collection/metabolic_disease_inherited Myoclonus

http://n.neurology.org/cgi/collection/myoclonus

Information about reproducing this article in parts (figures,tables) or in its entirety can be found online at:

http://www.neurology.org/about/about_the_journal\#permissions

Information about ordering reprints can be found online:

http://n.neurology.org/subscribers/advertise

Neurology ${ }^{\circledR}$ is the official journal of the American Academy of Neurology. Published continuously since 1951, it is now a weekly with 48 issues per year. Copyright @ 2018 American Academy of Neurology. All rights reserved. Print ISSN: 0028-3878. Online ISSN: 1526-632X.

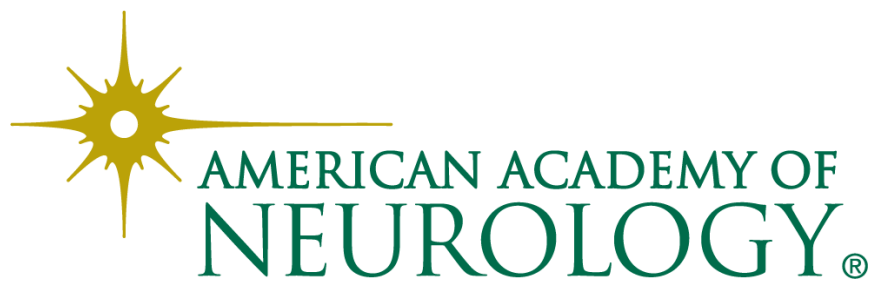

